\title{
A Rare Complication of Chylous Ascites Following Aortobifemoral Bypass Surgery
}

\author{
Görkem Yiğit' ${ }^{1}$, Alp Yildirim ${ }^{1}$, Mustafa Ozdemir ${ }^{2}$, Anil Ozen ${ }^{1}$ and Hakki Zafer Iscan ${ }^{1}$ \\ ${ }^{1}$ Department of Cardiovascular Surgery, Ministry of Health, Ankara City Hospital, Ankara, Turkey \\ ${ }^{2}$ Department of Radıology, Ministry of Health, Ankara City Hospital, Ankara, Turkey
}

\begin{abstract}
Injury of the lymphatic trunk during abdominal aortic surgery causes leakage of chyle into the peritoneum, resulting in chylous ascites. A 53-year male, who underwent aortobifemoral bypass surgery for Leriche syndrome in our clinic, presented to the Emergency Department two months postoperatively, with complaints of abdominal discomfort, reduced appetite and weight loss. Computed tomography imaging and ultrasound of the abdomen revealed a retroperitoneal fluid collection. The patient was admitted and treated with percutaneous drainage of the chylous ascites, total parenteral nutrition (TPN) of a low fatty diet containing medium-chain triglycerides (MCT), and intravenous antibiotics. He was discharged after three weeks of treatment. This case report is an example of a rare complication that may occur after abdominal aortic surgery; and enables us to review treatment options for the management of chylous ascites.
\end{abstract}

Key Words: Chyle, Ascites, Abdominal aortic surgery.

How to cite this article: Yiğit G, Yildirim A, Ozdemir M, Ozen A, Iscan HZ. A Rare Complication of Chylous Ascites Following Aortobifemoral Bypass Surgery. J Coll Physicians Surg Pak 2021; 31(07):849-851.

\section{INTRODUCTION}

The cisterna chyli can be injured during abdominal aortic surgery due to its close proximity to the abdominal aorta. Injury to this lymphatic sac causes leakage of chyle into the peritoneal cavity. Chyloperitoneum may present with various symptoms depending on the severity of the leakage. The most common symptoms are abdominal distension, dyspnea, weight loss and abdominal pain. In the literature to date, there are approximately 40 reports of patients who developed chylous ascites following aortic surgery. ${ }^{1}$

Chylous ascites, as a complication following abdominal aortic surgery, has a mortality rate of $11-18 \%^{2}$; and treatment can be very challenging. Here, we report a case of a 53 -year male developing chylous ascites following aortobifemoral bypass surgery; and discuss the treatment options.

\section{CASE REPORT}

A 53-year male underwent aortobifemoral bypass surgery for Leriche syndrome. On initial investigation, computed tomography angiography (CTA) revealed distal occlusion of the abdominal aorta, including the common iliac arteries and the internal iliac arteries.

Correspondence to: Dr. Görkem Yiğit, Department of Cardiovascular Surgery, Ministry of Health, Ankara City Hospital, Ankara, Turkey

E-mail: drgorkemyigit@gmail.com

Received: September 30, 2019; Revised: November 11, 2019; Accepted: November 26, 2019

DOI: https://doi.org/10.29271/jcpsp.2021.07.849
He had a history of hypertension, and was a heavy smoker. Conventional aortobifemoral bypass operation was performed. Following median laparatomy, the infrarenal aorta was clamped from $2-3 \mathrm{~cm}$ distal to the renal artery. The proximal anastomosis and the distal anastomoses were performed using a Dacron ${ }^{\circledR}$ graft in an end-to-side manner. There were neither any difficulties nor chylous leakage observed intraoperatively. The patient made an uneventful recovery and was discharged five days postoperatively. Two months postoperatively, the patient presented to the Emergency Department with complaints of abdominal discomfort, vomiting, reduced

appetite and weight loss of six kgs. CT and abdominal ultrasonography (USG) imaging revealed a retroperitoneal fluid collection $(5 \times 6 \mathrm{~cm})$ infrarenally around the superior mesenteric vein (SMV). The imaging of the fluid collection was reported as containing features compatible with an abscess that constricted the SMV (Figure 1). Routine blood tests revealed a white cell count within the normal range $\left(10.10 \times 10^{3} / \mathrm{uL}\right)$. The C-reactive protein (CRP) level was $120 \mathrm{mg} / \mathrm{L}$. The patient was hospitalised and piperacilin/tazobactam antibiotic therapy was started after blood cultures were taken. A catheter was placed where the chylous leakage had collected by an interventional radiologist and left in situ to aid the outward flow of chyle (Figure 2). Oral intake was stopped and total parenteral nutrition (TPN) therapy commenced. On the first day, 1,150 ml of milky and odourless chylous fluid was drained. A sample of the fluid was taken for culture and biochemical analysis. Chemical analysis of the chyle showed a total protein level of $15,400 \mathrm{mg} / \mathrm{L}$ and an albumin level of $2 \mathrm{mg} / \mathrm{L}$. Concurrent blood samples were taken for culture and biochemical analysis. The concentration of triglyceride was 369 
$\mathrm{mg} / \mathrm{dl}$ in the chylous fluid compared to $160 \mathrm{mg} / \mathrm{dl}$ in the blood. Culture of the chylous fluid revealed $E$. Coli sensitive to ertapenem. A diagnosis of infected chylous ascites was made and a continued treatment plan of antibiotics, 10 days of total parenteral hyperalimentation with no oral intake, and percutaneous drainage was decided upon. Follow-up CRP, and total protein and albumin levels were within the normal limits. The patient underwent a series of abdominal USG studies during the course of treatment. Once, the imaging revealed minimal remnants of the abscess with no accumulation of further abscess material, and the drain was removed. The patient was discharged three weeks after hospitalisation. A CT scan performed three months postoperatively, which showed no retroperitoneal collection.

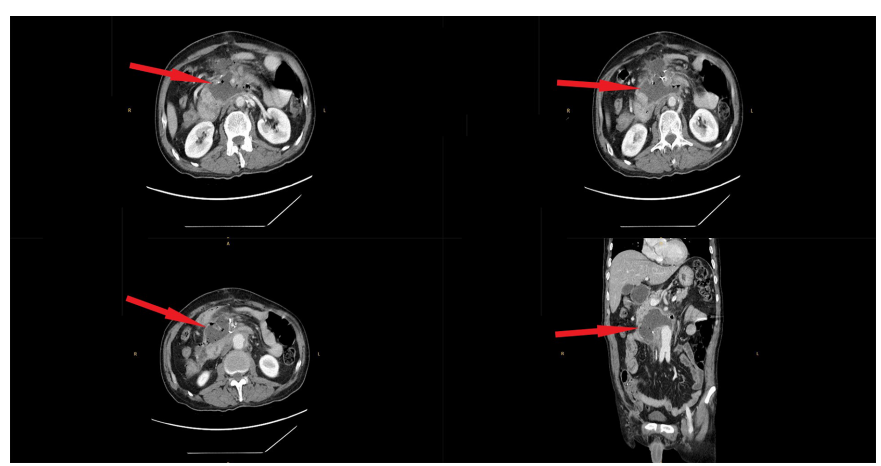

Figure 1: Retroperitoneal fluid collection $(5 \times 6 \mathrm{~cm})$ was detected by computed tomography and abdominal ultrasound anteriorly at the infrarenal level around the superior mesenteric vein (SMV).

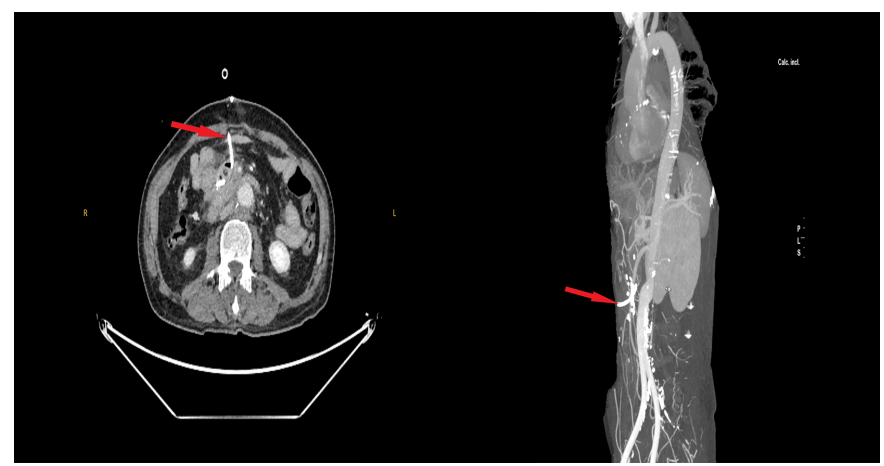

Figure 2: Catheter was placed in the area where chileous leakage was collected and left to flow continuously.

\section{DISCUSSION}

Chylous ascites is a serious complication of abdominal aortic surgery. Emergency surgery, such as ruptured abdominal aortic aneurysm and inflammatory aortic aneurysm surgery, have a higher risk of chylous complications. ${ }^{3,4}$ Injury to the cisterna chyli during surgical exploration may cause leakage of chylous fluid into the peritoneum and result in chyloperitoneum. Possibility of this complication should be kept in mind and the lymphatic ducts should be guarded during surgery. However, intraoperative visualisation of the lymphatic ducts may be difficult. Mortality rates of chylous complications due to abdominal aortic aneurysm surgery can be as high as $17 \%{ }^{5}$ Other surgical procedures that may cause chylous ascites are gastrectomy, nephrectomy, Wilm's tumor removal, duodenectomy and gynecological malignancy surgeries. ${ }^{6}$
The diagnosis of chylous ascites may be difficult because of its vague, non-specific symptoms. Chylous ascites develops within three to four weeks, following abdominal aortic surgery. ${ }^{7}$ The diagnosis is made using CT, USG and paracentesis. ${ }^{7}$ Paracentesis is the most sensitive diagnostic investigation. ${ }^{5}$ The paracentesis fluid material in most of the cases is characteristically milky. Chylous leakage has a sterile, odourless, milky apperance and possesses a triglyceride level greater than $200 \mathrm{mg} / \mathrm{dL}$, as was the case in this patient. ${ }^{1,5}$ The leakage of chylous material, loss of chylomicrons and leucocytes can cause multiple complications such as malnourishment, weight loss and immune deficiency. As a result, the patient in this case presented with complaints of reduced appetite, vomiting and excessive weight loss $(6 \mathrm{~kg})$.

The treatment of chylous ascites is quite challenging. The treatment aims to reduce lymphatic flow, so that lymphatic vessels have time to heal. Initial treatment should be conservative. The treatment consists of TPN or a low fat diet with MCT, somatostatin analogues and percutaneous drainage. TPN plays a significant role in reducing lymphatic leakage. ${ }^{8}$ MCTs can directly enter the portal circulation. A special MCT diet for decreasing bowel fat absorption is an essential component of lymphatic leakage treatment. ${ }^{3}$ Somatostatin analogues inhibit lymphatic flow and decrease intestinal blood flow. ${ }^{8}$ In this case, the combination of TPN and an MCT diet was preferred.

The surgical treatment option for chylous ascites should only be preferred if 4-6 weeks of conservative treatment fails or if the patient is elderly or an infant. This is because an untreated chylous leakage can be intolerable in these age groups. ${ }^{5}$ Lymphangiography should be done prior to surgery to identify potential major chylous leakage. The defect is then repaired laparoscopically with direct suture ligation or fibrin glue. If surgical treatment is not effective, a peritoneal-venous shunt or revision laparatomy can be used as the final line of treatment. In this case, conservative rather than surgical treatment was preferred due to the patient's age. Surgical treatment would have been considered, if the lympathic leakage had continued, thus worsening the patient's symptoms.

Chylous ascites is a rare complication of surgery. Nevertheless, it is essential to make a prompt, accurate diagnosis and to adopt a multidiciplinary approach to treatment, including input from surgeons, infectious diseases specialists, nutritionists and interventional radiologists. Early diagnosis may decrease high mortality rates. We believe that a systematic approach to treatment, starting with conservative treatment and a multidisciplinary approach is essential when treating these patients.

\section{PATIENT'S CONSENT:}

Informed consent was obtained from the patient to publish data regarding this case.

\section{CONFLICT OF INTEREST:}

The authors declared no conflict of interest.

\section{AUTHORS' CONTRIBUTION:}

GY, AY: Contributed to the conception and design of the work 
(evaluation of the case, detailed history taking, investigation and reporting the investigations).

MÖ, AÖ: Revised and critically analysed the content.

$\mathrm{HZI}$ : Finally approved the version.

\section{REFERENCES}

1. Galanopoulos G, Konstantopoulos T, Theodorou S, Tsoutsas I, Xanthopoulos D, Kaperonis E, et al. Chylous ascites following open abdominal aortic aneurysm repair: An unusual complication. Methodist Debakey Cardiovasc J 2016; 12(2): 119-21. doi: 10.14797/mdcj-12-2-119.

2. Pabst TS III, McIntyre KE Jr, Schilling JD, Hunter GC, Bernhard VM. Management of chyloperitoneum after abdominal aortic aneurysm. Am J Surg 1993; 166(2):198-9. doi: 10.1016/s0002-9610(05)81055-4.

3. Lv S, Wang Q, Zhao W, Han L, Wang Q, Batchu N, et al. A review of the postoperative lymphatic leakage. Oncotarget 2017; 8(40):69062-75. doi: 10.18632/oncotarget.17297.
4. Ching KC, Santos E, Mccluskey K, Jeyabalan G. CT-guided injection of $\mathrm{N}$-butyl cyanoacrylate glue for treatment of chylous leak after aorto-mesenteric bypass. Cardiovasc Intervent Radiol 2014; 37(4):1103-6. doi: 10.1007/ s00270-013-0811-z.

5. Duncan A. Local complications: Lymphatic. In: Rutherford's vascular surgery. 7th ed. 2010; pp. 722-723. Saunders.

6. Ohki S, Kurumisawa S, Misawa Y. Chylous ascites after abdominal aortic aneurysm repair. Ann Vasc Dis 2016; 9(1):48-50. doi: 10.3400/avd.cr.15-00098.

7. Uchinami M, Morioka K, Doi K, Nakamura T, Yoshida $M$, Tanaka $K$, et al. Retroperitoneal laparoscopic management of a lymphocele after abdominal aortic surgery: A case report. J VascSurg 2005; 42(3):552-5. doi: 10.1016/j.jvs.2005.04.029.

8. Olthof E, Blankensteijn JD, Akkersdijk G. Chyloperitoneum following abdominal aortic surgery. Vascular 2008; 16(5):258-62. doi: 10.2310/6670.2008.00035. 